\title{
IMAGEM CORPORAL DE ADOLESCENTES COM DEFICIÊNCIA VISUAL: UMA REVISÃO SISTEMÁTICA DE ESTUDOS SOBRE O TEMA
}

\author{
BODY IMAGE OF ADOLESCENTS WITH VISUAL \\ IMPAIRMENT: A SYSTEMATIC REVIEW OF STUDIES ON \\ THE THEME
}

\section{IMAGEN CORPORAL DE ADOLESCENTES CON DISCAPACIDAD VISUAL: UNA REVISIÓN SISTEMÁTICA DE LOS ESTUDIOS SOBRE EL TEMA}

\begin{abstract}
Augusta Quintanilha ${ }^{1}$
https://orcid.org/0000-0003-3680-6674

Paulo Gutierres Filho ${ }^{2}$

https://orcid.org/0000-0001-7753-0825

Clara Mockdece Neves ${ }^{3}$

https://orcid.org/0000-0002-5240-6710

Juliana Fernandes Filgueiras Meireles ${ }^{4}$

https://orcid.org/0000-0001-8396-6449

Maria Elisa Caputo Ferreira ${ }^{5}$

https://orcid.org/0000-0002-3294-7560

Ravine Carvalho Pessanha Coelho da Silva ${ }^{6}$

https://orcid.org/0000-0002-1537-8941
\end{abstract}

Vitor Alexandre Rabelo de Almeida ${ }^{7}$

https://orcid.org/0000-0001-7082-7490

Fabiane Frota da Rocha Morgado ${ }^{8}$

https://orcid.org/0000-0002-3969-9029

\footnotetext{
${ }^{1}$ Universidade Federal Rural do Rio de Janeiro, Seropédica, Rio de Janeiro - Brasil. E-mail: augustapsic@gmail.com.

${ }^{2}$ Universidade de Brasília, Brasília, Distrito Federal - Brasil. E-mail: profgutierres@gmail.com.

3 Universidade Federal de Juiz de Fora, Juiz de Fora, Minas Gerais - Brasil. E-mail: clarinhamockdece@hotmail.com.

${ }^{4}$ Kennesaw State University, Georgia - USA. E-mail: eujuly90@ hotmail.com.
} 
QUINTANILHA, A.; GUTIERRES FILHO, P.; NEVES, C. M.; MEIRELES, J. FERREIRA, M. E. C.; SILVA, R. C. P. C. da; ALMEIDA, V. A. R. de; MORGADO, F. F. da R.

5 Universidade Federal de Juiz de Fora, Juiz de Fora, Minas Gerais - Brasil. E-mail: caputoferreira@terra.com.br.

${ }^{6}$ Universidade Federal Rural do Rio de Janeiro, Seropédica, Rio de Janeiro - Brasil. E-mail: ravinecarvalho@hotmail.com.

${ }^{7}$ Universidade Federal Rural do Rio de Janeiro, Seropédica, Rio de Janeiro - Brasil. E-mail: vitoralexandrea_@hotmail.com.

${ }^{8}$ Universidade Federal Rural do Rio de Janeiro, Seropédica, Rio de Janeiro - Brasil. E-mail: fabi.frm@hotmail.com.

\title{
Resumo
}

A imagem corporal é um constructo amplamente estudado e pesquisas nessa área têm aumentado nos últimos anos, entretanto adolescentes com deficiência visual ainda carecem de investigação. Esta revisão sistemática tem por objetivo investigar trabalhos que buscam evidências científicas sobre aspectos da imagem corporal de adolescentes com deficiência visual. Realizou-se uma busca sistemática de artigos em quatro bases de dados, Scopus, PsycInfo, PubMed e Web of Science. O recrutamento dos estudos, sem restrição temporal, foi concluído em 8 de junho de 2019. Foram incluídos 32 artigos, os quais se classificaram em quatro categorias: (1) aspectos psicossociais do corpo, (2) conceito corporal, (3) atividade física/saúde (4) instrumentos de avaliação da imagem corporal e insatisfação corporal. A maioria dos estudos inseridos nesta revisão demonstraram, mediante evidências científicas, que há implicações negativas sobre a imagem corporal de adolescentes com deficiência visual, Alguns achados, contudo, baseiam-se nas limitações da deficiência, valorizando o déficit orgânico. Fazem-se necessários estudos que busquem uma abordagem biopsicossocial na formação da imagem corporal positiva nesta população.

Palavras-chaves: Autoconceito. Autoestima. Imagem Corporal. Educação. Cegueira.

\begin{abstract}
Body image is a widely studied construct and research in this area has grown in recent years. Nevertheless, adolescents with visual impairment need to be investigated. This systematic review aims to investigate studies that seek scientific evidence on the body image of adolescents with visual impairment. A systematic search of articles was carried out in four databases: Scopus, PsycInfo, PubMed and Web of Science. The recruitment of studies, without time restriction, was completed on June 8th, 2019. A total of 32 articles were included, which were carefully analyzed and classified into 4 categories: (1) psychosocial aspects of the body, (2) body concept, (3) physical activity/health (4) instruments for assessing body image and body dissatisfaction. Most of the studies included in this review demonstrated through scientific evidence that there are negative implications for the body image of adolescents with visual impairment, however, some findings are based on the limitations of the impairment, valuing the organic deficit. Studies that seek a biopsychosocial approach in the formation of positive body image in this population are necessary.
\end{abstract}

Keywords: Self-concept. Self-esteem. Body Image. Education. Blindness.

\section{Resumen}

La imagen corporal es una construcción ampliamente estudiada y la investigación en esta área ha crecido en los últimos años. Sin embargo, adolescentes con discapacidad visual aún 
necesitan ser investigados. Esta revisión sistemática tiene como objetivo investigar estudios que busquen las evidencias científicas sobre la imagen corporal de los adolescentes con discapacidad visual. Se realizó una búsqueda sistemática de artículos en cuatro bases de datos, Scopus, PsycInfo, PubMed y Web of Science. La contratación de estudios, sin restricción de tiempo, se completó el 8 de junio de 2019. Se incluyeron 32 artículos, que se analizaron cuidadosamente y se les clasificaron en cuatro categorías: (1) aspectos psicosociales del cuerpo, (2) concepto del cuerpo, (3) actividad física/salud y (4) instrumentos para evaluar la imagen corporal y la insatisfacción corporal. La mayoría de los estudios incluidos en esta revisión demostró, a través de evidencia científica, que existen implicaciones negativas para la imagen corporal de los adolescentes con discapacidad visual. Sin embargo, algunos hallazgos se basan en las limitaciones de la discapacidad, valorando el déficit orgánico. Los estudios que buscan un enfoque biopsicosocial en la formación de una imagen corporal positiva en esta población son necesarios.

Palabras clave: Autoconcepto. Autoestima. Imagen Corporal. Educación. Ceguera.

\section{Introdução}

Deficiência visual consiste em qualquer alteração no sistema visual que pode levar a baixa visão ou cegueira (BOURNE et al., 2017; RIBEIRO, 2017; OTTAIANO et al., 2019) e tem sido conceituada a partir de diferentes perspectivas, entre as quais se destacam a médica, a social e a biopsicossocial. A primeira avalia a deficiência visual com viés pautado nas limitações e incapacidades do sujeito acometido (GZIL et al., 2007; BISOL; PEGORINI; VALENTINI, 2017), enquanto, na abordagem social, esse tipo de deficiência é considerado uma construção social (FRANÇA, 2013; GAUDENZI; ORTEGA, 2016). A perspectiva biopsicossocial aborda a deficiência visual como um fenômeno multidimensional, simultaneamente construído no corpo e nas relações sociais (JENKS, 2005; MORGADO et al., 2017).

$\mathrm{Na}$ esteira da perspectiva biopsicossocial, uma área de estudos que se destaca é a imagem corporal. Esse constructo é considerado a figuração do corpo representada na mente, sendo responsável pelo conhecimento que se tem acerca de si mesmo (SCHILDER, 1994). O desenvolvimento da imagem corporal é, portanto, um constructo multidimensional, que engloba elementos da percepção corporal, como estimativa dos aspectos físicos do corpo e de suas funções e elementos atitudinais, como valorização e estima do corpo (THOMPSON; BURKE; KRAWCZYK, 2012; CASH, 2004). O desenvolvimento da imagem corporal pode ir tanto por um caminho positivo, configurando a imagem corporal positiva - atitudes de afeto, amor e respeito para com o próprio corpo (TYLKA; BARCALOW, 2015) - quanto em 
QUINTANILHA, A.; GUTIERRES FILHO, P.; NEVES, C. M.; MEIRELES, J. FERREIRA, M. E. C.; SILVA, R. C. P. C. da; ALMEIDA, V. A. R. de; MORGADO, F. F. da R.

um sentido negativo, caracterizando a imagem corporal negativa - desgosto profundo com o próprio corpo ou com a aparência corporal (MORGADO et al., 2013).

Quando o enfoque é sobre adolescentes com deficiência visual, a temática da imagem corporal assume especial relevância. A adolescência é a fase da vida caracterizada entre 10 e 19 anos (WHO, 2009) na qual o indivíduo passa por alterações em aspectos biológicos, cognitivos, psicológicos e sociais (MÄIKINEN, 2015; BLAKEMORE; MILLS, 2014; KAPLAN, 2000). Alguns estudiosos apontam que essas pessoas podem apresentar dificuldades em desenvolver e manter uma imagem corporal positiva, configurando um importante grupo de risco (HALDER; DATTA, 2011; PINQUART; PFEIFFER, 2012; GÜLER et al., 2012). Há também registros que destacam o desenvolvimento de imagem corporal negativa nessa população, e isso tanto pela dificuldade de fazer observações tácteis do corpo dos outros (VANDEREYCKEN, 1986) como também por fatores relacionados com a limitada prática de atividade física (ANDERSON et al., 2019; HAEGELE; ZHU, 2018; CHEIKH; KASMI; MEHIDI, 2018; WRZESINSKA et al., 2016).

Destaca-se ainda que a deficiência visual pode estar associada à imagem corporal em diferentes contextos, pois estudos demonstram que adolescentes com algum grau de comprometimento visual podem ser acometidos por quadros de anorexia nervosa (VANDEREYCKEN, 1986), depressão (KOCOURKOVA et al., 2011), insatisfação corporal (ASHIKALI; DITTIMAR, 2010; HALDER; DATTA, 2011), exclusão social (HAEGELE; ZHU, 2018), checagem corporal (GÜLER et al., 2012) e imagem corporal negativa (GREGUOL; GOBBI; CARRARO, 2014).

Nesse sentido, é essencial conhecer de modo sistemático estudos que têm investigado a imagem corporal de adolescentes com deficiência visual, de modo a ampliar a compreensão nessa área e viabilizar intervenções mais assertivas e eficazes no contexto educacional e da saúde. Em adição, esse conhecimento pode prover informações a respeito de processos cognitivos-emocionais que desencadeiam a imagem corporal positiva, bem como alterações psicossociais que contribuem para o quadro de imagem corporal negativa. Assim, esta revisão sistemática tem por objetivo investigar trabalhos que buscam evidências científicas sobre aspectos da imagem corporal de adolescentes com deficiência visual. 


\section{Método}

\subsection{Estratégia de pesquisa}

Para esta revisão sistemática, foram identificados 2.529 artigos das bases de dados Scopus, PsycInfo, PubMed e Web of Science. Não houve restrição de data na busca da literatura, que se iniciou em 1 de junho de 2019 e terminou em 8 de junho do mesmo ano. Os descritores para essa pesquisa foram: ("visually impairment" OR "acquired blind” OR "acquired blindness" OR "congenital blindness" OR "visual impairment" OR "blind*" OR "congenital blind" OR "low vision" OR "visual disability") AND ("adolescent*” OR "teenagers" OR "students" OR "school children") AND ("body image") em todos os campos. Três revisores avaliaram os artigos de forma independente. As discordâncias na elegibilidade dos artigos foram resolvidas de forma consensual entre os revisores. Esta revisão sistemática foi registrada na Internacional Prospective of Systematic Reviews (PROSPERO) sob o número CRD42020144958. Também foram seguidas as recomendações propostas pelo Preferred Reporting Itens for Systematic Reviews and Meta-analyse: The Prisma Statement (MOHER et al., 2008).

Foram incluídos artigos em português, inglês e espanhol relacionados com a imagem corporal de adolescentes entre 10 e 19 anos de idade com deficiência visual. Também foram considerados estudos que utilizaram somente uma parcela dessa faixa etária, como, por exemplo, pesquisas com a população entre 18 e 40 anos (ASHIKALI; DITTIMAR, 2010; HAEGELE; ZHU, 2018). Foram excluídos artigos duplicados nas bases de dados analisadas, capítulos de livros, artigos de revisão e artigos que não estivessem associados a imagem corporal, adolescência e deficiência visual. Extraíram-se de cada estudo os seguintes dados: nome dos autores, ano, método de pesquisa, medidas utilizadas, país no qual o estudo foi conduzido, população, objetivo, conclusão e qualidade dos artigos. Para avaliação dos dados, utilizou-se a análise descritiva e de conteúdo categorial de Bardin (2016), que classifica elementos que constituem um conjunto, reagrupando-os em razão da similaridade do conteúdo.

\subsection{Qualidade dos artigos}

Para avaliar a qualidade dos artigos foi utilizada a Quality Assessment Tool (QATSDD) (SIRRIYEH et al., 2012). Foram usados os 16 indicadores dessa ferramenta, pois 
QUINTANILHA, A.; GUTIERRES FILHO, P.; NEVES, C. M.; MEIRELES, J. FERREIRA, M. E. C.; SILVA, R. C. P. C. da; ALMEIDA, V. A. R. de; MORGADO, F. F. da R.

este estudo abarca pesquisas quantitativas, qualitativas e de métodos mistos. Os indicadores foram classificados, subjetivamente, em uma escala Likert de 4 pontos graduada de 0 a 3. Dessa forma, a pontuação pode alcançar o máximo de 48 pontos (quando a pesquisa é de método misto). Pontuações mais altas indicam artigos de maior qualidade. Artigos com pontuação igual ou superior a 50\% foram classificados com qualidade boa ou elevada. Em contrapartida, estudos que não atingiram essa pontuação foram considerados de qualidade inferior ao esperado (SIRRIYEH et al., 2012). Entretanto, mesmo com pontuação inferior a $50 \%$, tais artigos não foram excluídos pelo critério de qualidade. A Figura 1 mostra o fluxograma resumindo a estratégia adotada para identificação e seleção dos estudos:

Figura 1 - Fluxograma do processo sistemático de identificação e seleção dos artigos.

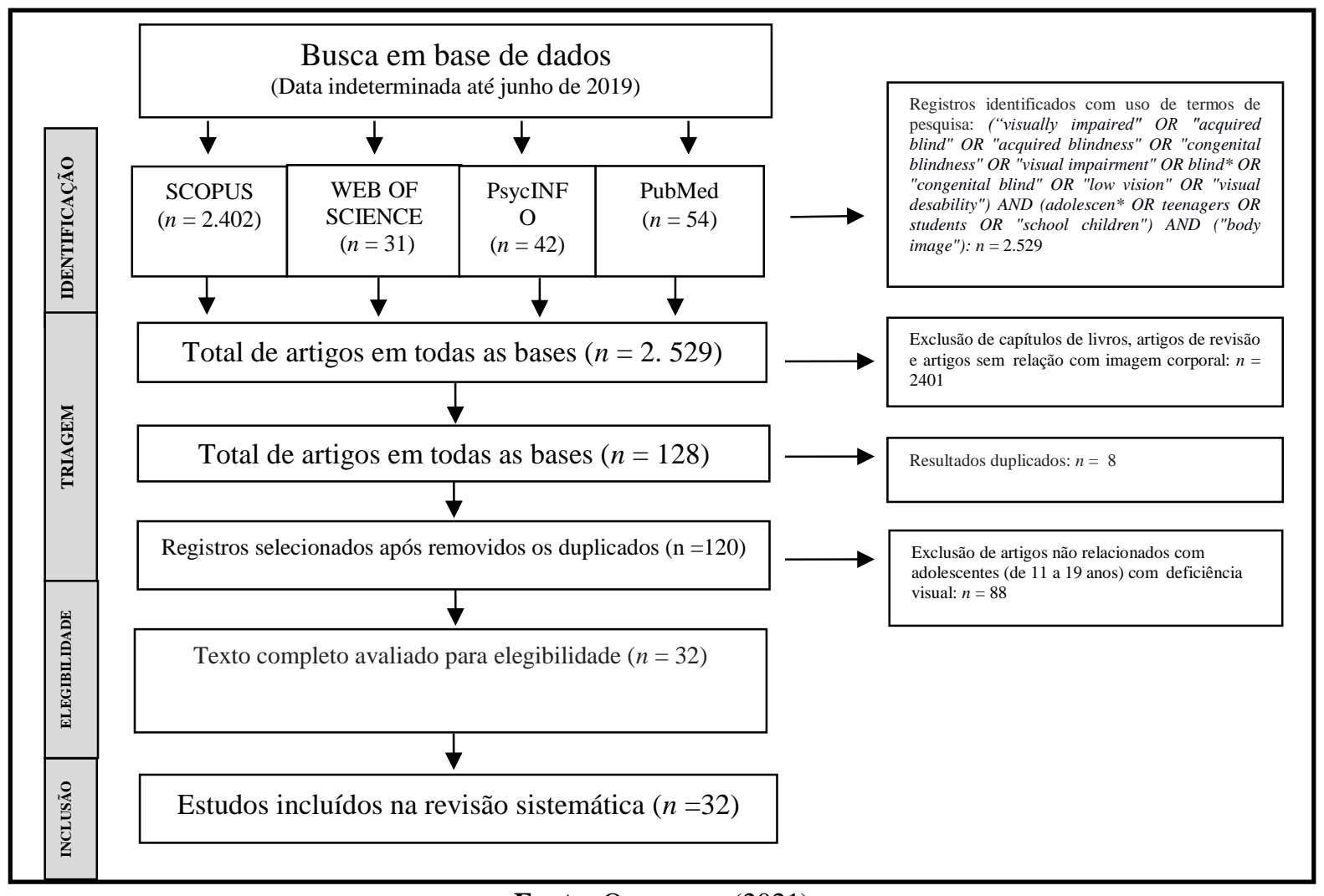

Fonte: Os autores (2021)

\section{Resultados}

Foram identificados, inicialmente, 2.529 artigos, dos quais 32 foram incluídos. Na análise foi possível observar que a maioria $(n=24,76,5 \%)$ foi publicada entre os anos de 
2009 e 2019, demonstrando um expressivo aumento do interesse em estudar essa temática na última década, conforme pode ser observado na Figura 2.

Figura 2 - Evolução cronológica dos estudos incluídos nessa revisão.

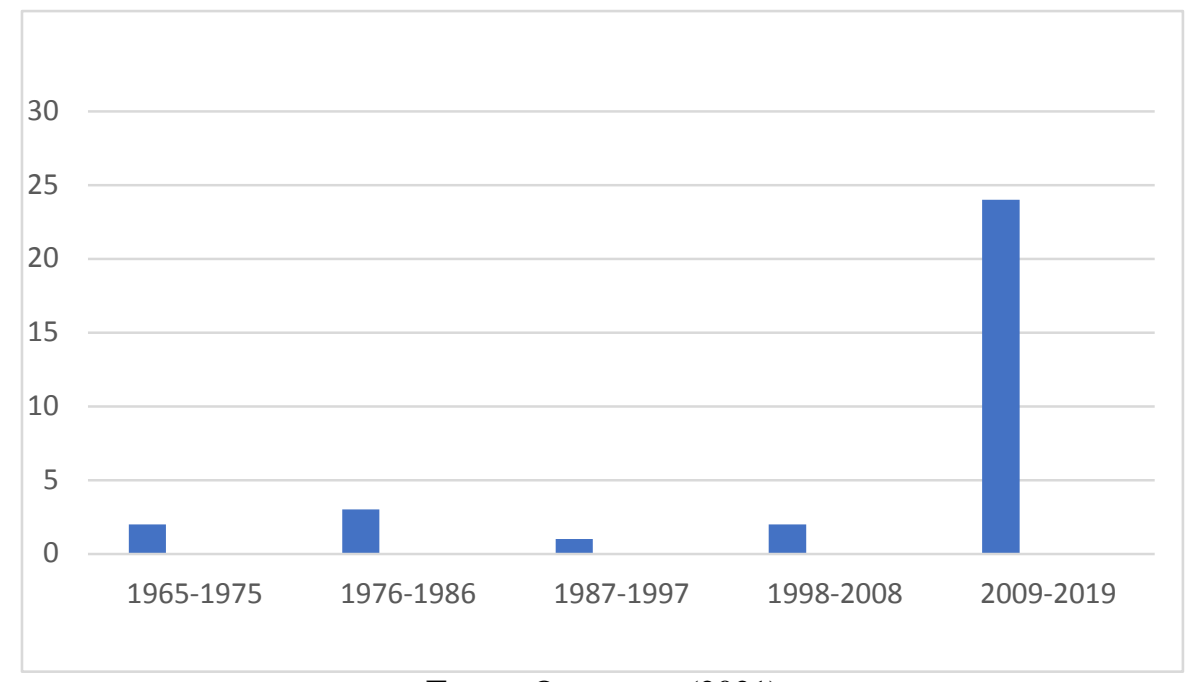

Fonte: Os autores (2021)

O primeiro estudo publicado data de 1968 (WITKIN et al., 1968), enquanto o último, de 2019 (ANDERSON et al., 2019). Os países com maior número de publicações foram Estados Unidos ( $n=6 / 19 \%)$, Brasil e Grécia ( $n=4 / 12 \%$ para ambos), Alemanha e Austrália ( $n=3 / 9 \%$, para ambos) e outros países $(n=12 / 38 \%)$.

Em relação à amostra, a maioria dos estudos analisados reportou incluir participantes adolescentes com deficiência visual, sem especificar o grau de acometimento da visão ( $n=$ $22 / 69 \%$ ), todavia outros estudos especificaram claramente trabalhar apenas com adolescentes com cegueira $(n=10 / 31 \%)$. Grande parte dos artigos desta revisão sistemática incluiu ambos os sexos ( $n=22 / 69 \%$ ), entretanto houve artigos que estudaram especificamente o sexo feminino $(n=6 / 19 \%)$, o sexo masculino $(n=2 / 6 \%)$ e outros ainda que não determinaram o $\operatorname{sexo}(n=2 / 6,25 \%)$.

Outro achado relevante aponta que os estudos analisados possuem métodos N quantitativos e qualitativos, incluindo estudos transversais $(n=25 / 71,42 \%)$, longitudinais $(n$ $=4 / 12,5 \%)$ e estudos de caso $(n=3 / 9,3 \%)$. Maiores detalhamentos sobre os estudos analisados podem ser visualizados na Tabela 1, disponibilizada em arquivo suplementar. 
QUINTANILHA, A.; GUTIERRES FILHO, P.; NEVES, C. M.; MEIRELES, J. FERREIRA, M. E. C.; SILVA, R. C. P. C. da; ALMEIDA, V. A. R. de; MORGADO, F. F. da R.

A respeito dos estudos que trazem evidências científicas sobre a imagem corporal de adolescentes com deficiência visual, a análise de conteúdo dos dados aponta a formação de quatro categorias temáticas distintas, agrupadas pela semelhança em seus conteúdos: a) aspectos psicossociais do corpo - agrupa estudos que abordam aspectos sociais, bem como emoções e afetos relacionados com o corpo; b) conceito corporal - congrega estudos que se referem a como o adolescente com deficiência visual percebe seu corpo no espaço e o utiliza nas mais variadas funções; c) atividade física/saúde - abrange artigos que estudam a importância da atividade física para os adolescentes com deficiência visual na formação de sua imagem corporal; d) instrumentos de avaliação da imagem corporal e insatisfação corporal - abarcam aspectos relacionados com as escalas de imagem corporal para adolescentes com deficiência visual e insatisfação com o corpo. A Figura 3 detalha a formação dessas categorias.

Figura 3 - Distribuição dos estudos pela análise de conteúdo categórica.

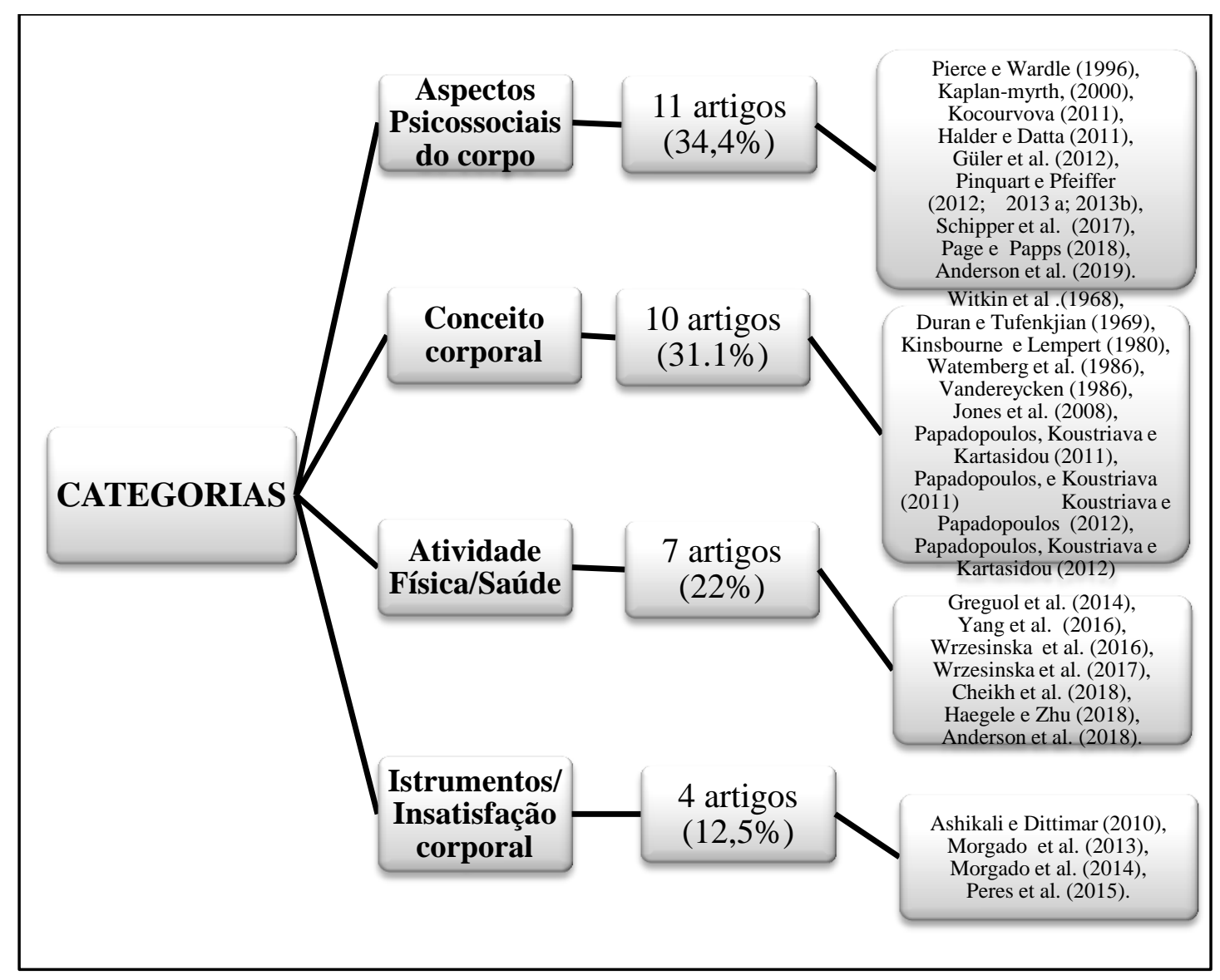

Fonte: Os autores (2021) 


\section{Discussão}

A presente revisão sistemática tem por objetivo investigar trabalhos que buscam as evidências científicas sobre a imagem corporal de adolescentes com deficiência visual. Os resultados apontam a configuração de quatro grandes categorias, que serão discutidas em tópicos após a discussão das características gerais dos estudos analisados em relação a: (1) evolução cronológica dos estudos, (2) países de realização dos estudos, (3) características da amostra (tipo de deficiência e sexo estudado), (4) métodos dos estudos realizados e (5) qualidade dos estudos.

No que tange à evolução cronológica, a presente revisão sistemática demonstrou que, desde a década de 1960, pesquisadores têm buscado compreender melhor a deficiência visual e as implicações que a sociedade trás na formação da imagem corporal, todavia a maioria dos estudos se deu na última década $(n=24 / 76 \%)$, demonstrando aumento expressivo nesse período. Esses dados se coadunam com achados sobre o aumento de estudos sobre a imagem corporal nas mais diversas populações (FARHAT; IANNOTTI; CACCAVALE, 2014; LAUS et al., 2014; NEVES; MORGADO; TAVARES, 2015; TREMBLAY et al., 2016; SOULLIARD; WAL, 2019).

A respeito dos locais de realização dos estudos, os Estados Unidos foram o país com mais investigações sobre imagem corporal em adolescentes com deficiência visual $(n=6)$, contudo a metade dos artigos nesse país foi publicada até a década de 1980 (WITKIN et al., 1968; DURAN; TUFENKJIAN, 1969; WATEMBERG; SERMARK; HENDERSON, 1986). Somente três desses estudos são mais recentes (JONES; TAYLOR; BROADWELL, 2008; SCHIPPER; LIEBERMAN; MOODY, 2017; HAEGELE; ZHU, 2018), demonstrando a necessidade de pesquisas atualizadas sobre a temática nesse país com tamanho potencial de publicação nas mais diferentes áreas do saber (FERNANDEZ et al., 2015; STAMER; SCHUMAN, 2017; JALALI et al., 2019). Em seguida, Brasil e Grécia aparecem como os países com mais publicações na área. No Brasil, o interesse por essa temática vai ao encontro do aumento de estudos gerais na área da imagem corporal, já apontado por Neves, Morgado e Tavares (2015). Concernente à Grécia, os estudos relacionados com a imagem corporal têm se ampliado nos últimos anos (BONOTIS et al., 2016), inclusive na adaptação transcultural de instrumentos de avaliação da imagem corporal (ARGYRIDES; KKHELI; KENDEOU, 2014; ALEXIAS; TOGAS; MELLON, 2016). 
QUINTANILHA, A.; GUTIERRES FILHO, P.; NEVES, C. M.; MEIRELES, J. FERREIRA, M. E. C.; SILVA, R. C. P. C. da; ALMEIDA, V. A. R. de; MORGADO, F. F. da R.

Quanto ao tipo de deficiência, constatou-se que uma parte dos estudos comparou adolescentes com deficiência visual e videntes (WITKIN et al., 1968; KINSBOURNE; LEMPERT, 1980; WATEMBERG; SERMARK; HENDERSON, 1986; ASHIKALI; DITTIMAR, 2010; HALDER; DATTA, 2011; PINQUART; PFEIFFER, 2012; PINQUART; PFEIFFER, 2013a; CHEIKH; KASMI; MEHIDI, 2018). Essa constatação viabiliza reflexões sobre processos normativos da deficiência visual. De acordo com Morgado et al. (2019), avaliar pessoas com deficiência visual tendo como parâmetro videntes não parece ser adequado na análise da imagem corporal, visto que ambos os grupos apresentam formas diferenciadas de obter informações do próprio corpo na sua relação com o mundo.

A maioria dos artigos $(n=21)$ utilizou ambos os sexos em sua amostra (WITKIN et al., 1968; DURAN; TUFENKJIAN, 1969; PIERCE; WARDLE, 1996; KAPLAN-MYRTH, 2000; HALDER; DATTA, 2011; PAPADOPOULOS; KOUSTRIAVA, 2011; PAPADOPOULOS; KOUSTRIAVA; KARTASIDOU, 2011; KOUSTRIAVA; PAPADOPOULOS, 2012; PAPADOPOULOS; KOUSTRIAVA; KARTASIDOU, 2012; PINQUART; PFEIFFER, 2012; PINQUART, PFEIFFER, 2013a; 2013b; MORGADO et al., 2013; GREGUOL; GOBBI; CARRARO, 2014; MORGADO; CAMPANA; TAVARES, 2014; PERES et al., 2015; YANG et al., 2016; WRZESINSKA et al., 2016; SCHIPPER; LIEBERMAN; MOODY, 2017; WRZESINSKA et al., 2017; ANDERSON et al., 2018). Estudos que compõem a amostra com ambos os sexos possibilitam a comparação na formação da imagem corporal entre meninos e meninas com deficiência visual, além de considerarem a singularidade de cada um desses grupos no desenvolvimento de sua imagem corporal. De acordo com Pinquart e Pfeifer (2012), no que tange a adolescentes com deficiência visual, meninos são mais propícios a desenvolver e manter a imagem corporal positiva do que meninas. Achados similares foram observados entre adolescentes meninas videntes (JONES, 2004; DAVISON; MCCABE, 2006, ABBOTT; BARBER, 2010), todavia encontram-se controvérsias nesses achados, pois adolescentes videntes de ambos os sexos podem apresentar insatisfação corporal (HAYNOS et al., 2019).

Considerando as características metodológicas, a maior parte das pesquisas encontradas foi transversal, o que demonstra que a aplicação de uma pesquisa num recorte específico pode prover uma visão geral sobre um fenômeno; todavia estudos transversais possuem limitações, pois não permitem estabelecer relações causais (MORGADO et al., 2017). Estudos longitudinais também foram encontrados, demonstrando a importância da continuidade de pesquisas com essa população. Estudos de caso foram a minoria e os 
resultados desse método de pesquisa devem ser observados com cuidado, evitando generalizações (MORGADO et al., 2019).

No que tange à qualidade dos artigos, as pontuações foram entre 9 e 48 pontos. Apenas um artigo (MORGADO; CAMPANA; TAVARES, 2014) atingiu a pontuação máxima de 48 pontos no QATSDD. Em 72\% dos artigos $(n=23)$, a pontuação ficou entre 21 e 48 pontos (acima da média), demonstrando uma boa qualidade na pesquisa, enquanto $28 \%$ $(n=9)$ obtiveram pontuação entre 9 e 20 pontos (abaixo da média), sendo considerados artigos de baixa qualidade. Entre os estudos, 46\% $(n=5)$ forneceram evidências que recrutaram uma amostra representativa da população e 50\% $(n=16)$ relataram minuciosamente os procedimentos de coleta de dados. Em ambos os aspectos, esses artigos atingiram uma pontuação máxima de 3 pontos para o critério. Considerando as evidências científicas sobre a imagem corporal de adolescentes com deficiência visual, os resultados serão discutidos em quatro categorias formuladas no presente estudo em tópicos.

\subsection{Aspectos psicossociais do corpo}

Os aspectos psicossociais do corpo se referem ao desenvolvimento da imagem corporal com relação aos aspectos sociais, ou seja, o quanto os valores sociais influenciam o sujeito na percepção do próprio corpo e na formação da imagem corporal (THOMPSON et al., 2004; JUDITH; CAROL, 2006).

$\mathrm{Na}$ presente categoria, parte dos estudos demonstrou evidências científicas de que a deficiência visual pode ter implicações negativas (KAPLAN-MYRTH, 2000; HALDER; DATTA, 2011; KOCOURVOVA et al., 2011; GÜLER et al., 2012; PINQUART; PFEIFFER, 2012; 2013a; 2013b; PAGE; PAPPS, 2018) ou positivas (PIERCE; WARDLE, 1996; SCHIPPER et al., 2017; ANDERSON et al., 2018) na formação da imagem corporal em adolescentes.

Foram observados fatores como influência do contexto social na formação da imagem corporal dessas pessoas (HALDER; DATTA, 2011; KOCOURVOVA et al., 2011). Essas influências estão ligadas a aspectos como opinião dos pais na formação da autoestima (PIERCE; WARDLE, 1996) e como a disponibilidade parental e as conexões sociais podem refletir positivamente na formação da imagem corporal (PINQUART; PFEIFFER, 2013a). 
QUINTANILHA, A.; GUTIERRES FILHO, P.; NEVES, C. M.; MEIRELES, J. FERREIRA, M. E. C.; SILVA, R. C. P. C. da; ALMEIDA, V. A. R. de; MORGADO, F. F. da R.

Há, ainda, registros de que esses adolescentes se preocupam com a aparência física, pois, embora não possam se ver, outras pessoas podem vê-los. Essa preocupação pode levar a alterações na imagem corporal (KAPLAN-MYRTH, 2000). Halder e Datta (2011), por exemplo, reportaram que adolescentes com deficiência visual têm menores níveis de autoconceito e satisfação corporal quando em comparação com videntes.

Outra questão que chama a atenção se refere a transtornos alimentares que têm sido identificados entre adolescentes com deficiência da visão. Essas pessoas podem desenvolver compulsão alimentar, preocupação excessiva com a imagem corporal, comportamento de checagem corporal e dificuldades nas relações sociais. Esses aspectos podem estar relacionados com preocupações com sobrepeso e a opinião alheia, contudo o suporte familiar é fundamental no processo terapêutico (KOCOURVOVA et al., 2011).

É importante mencionar que a estimulação auditiva e tátil ajuda na percepção dos pares e do espaço, pois usam os canais auditivo e tátil para desenvolver conceitos sobre imagem corporal. A perda visual progressiva, entretanto, pode influenciar a formação da imagem corporal, visto que, ao serem utilizadas expressões como "vejo minhas pernas gordas", percebem-se resquícios da visão na descrição da imagem corporal (GÜLER et al., 2012).

Pinquart e Pfeiffer (2012) relataram que adolescentes do sexo feminino com deficiência visual podem desenvolver uma imagem corporal negativa por serem dependentes da avaliação dos outros sobre sua aparência física. Esses mesmos autores destacaram que adolescentes com cegueira diferiram daqueles com baixa visão com relação ao desenvolvimento de identidade, demonstrando que a cegueira pode limitar a aquisição de informações sobre diferentes maneiras de viver (PINQUART; PFEIFFER, 2013a). Esses estudos ilustram que a deficiência da visão pode desencadear problemas psicossociais, contribuindo para o desenvolvimento e a manutenção da imagem corporal negativa.

Em consonância com esses achados, Pinquart e Pfeiffer (2013b) destacaram que, quanto maior a disponibilidade parental, maior a satisfação com a vida, indicando que escolas especiais para adolescentes com deficiência visual reduzem a disponibilidade de apoio dos pais, o que pode ajudar na melhora do autoconceito e da autoestima. No que tange à internalização de padrões sociais, Page e Papps (2018) citaram que tais padrões podem interferir diretamente no desenvolvimento de uma imagem corporal negativa. Como consequência, podem desenvolver desordens alimentares nesse público. Outros estudos 
obtiveram achados nesse mesmo sentido, apontando que a deficiência visual pode favorecer alterações negativas na imagem corporal entre adolescentes e aumentar o isolamento social, desencadeando problemas no desenvolvimento e na manutenção de uma imagem corporal positiva (KAPLAN-MYRTH, 2000; GREGUOL; GOBBI; CARRARO, 2013).

Embora os registros citados destaquem implicação negativa da deficiência visual na imagem corporal, a literatura é contraditória. Para Pierce e Wardle, (1996), adolescentes com deficiência visual experimentam aspectos afetivos relacionados com o corpo de acordo com a avaliação dos pais, refletindo em sua autoestima. Os autores relataram ainda que esses adolescentes são capazes de apresentar uma imagem corporal integrada, não baseada somente na aparência, o que lhes possibilita ampliar as relações saudáveis e positivas estabelecidas com o corpo.

Semelhante a esses achados, Schipper, Lieberman e Moody (2017) demonstraram que adolescentes com deficiência da visão têm uma percepção positiva sobre o que podem fazer. Meninos relataram não se importar com a aparência, porém meninas foram mais sensíveis à opinião dos outros. Da mesma forma, Anderson et al. (2019) reportaram que os elementos importantes para essa população eram os mesmos valorizados pela maioria das pessoas, entretanto os vivenciavam de forma diferente.

$\mathrm{Na}$ compreensão dos estudos agrupados nessa categoria, destaca-se que a imagem corporal não deve ser entendida somente como um conceito cognitivo com base na habilidade visual, mas como um constructo mental que pode ser influenciado por interpretações da realidade e valores socioculturais (THOMPSON, 2004; KOCOURVOVA et al., 2011). Em seguida, buscou-se entender a deficiência visual relacionada com a formação do conceito corporal em adolescentes.

\subsection{Conceito corporal}

A presente categoria apresenta estudos que abordam a formação do conceito corporal em adolescentes com deficiência visual. Os artigos investigaram como os adolescentes utilizam seu corpo como instrumento de medida para conhecer o ambiente e no conhecimento de si, favorecendo, assim, a formação de sua imagem corporal. Destaca-se que esses artigos faziam tanto referência à imagem corporal em uma perspectiva negativa (KINSBOURNE; LEMPERT, 1980; WATEMBERG; SERMARK; HERDERSON, 1986; VANDEREYCKEN, 1986; PAPADOPOULOS; KOUSTRIAVA, 2011; PAPADOPOULOS; KOUSTRIAVA; 
QUINTANILHA, A.; GUTIERRES FILHO, P.; NEVES, C. M.; MEIRELES, J. FERREIRA, M. E. C.; SILVA, R. C. P. C. da; ALMEIDA, V. A. R. de; MORGADO, F. F. da R.

KARTASIDOU ,2011, 2012) como positiva (WITKIN et al., 1968; DURAN; TUFENKJIAN, 1969; JONES; TAYLOR; BROADWELL, 2008; KOUSTRIAVA; PAPADOPOULOS, 2012), demonstrando inconsistência teórica na área.

Kinsbourne e Lempert (1980), por exemplo, constataram que adolescentes com deficiência visual têm a representação do corpo humano mais empobrecida e desproporcional em comparação com adolescentes videntes e adolescentes vendados, contudo utilizam o tato para construir o esquema corporal. Nesse mesmo sentido, Vandereycken (1986) reportou que adolescentes que não enxergam precisam primordialmente tocar o corpo para a formação da imagem corporal, o que se torna difícil quando necessitam transpor essas referências para fora do próprio corpo na construção de alguns conceitos como lateralidade, uma vez que precisam tocar a si e o outro. Nessa perspectiva, Watemberg, Sermark e Henderson (1986) constataram que adolescentes com deficiência visual desenvolvem conceitos de esquema corporal por meio do sentido cinestésico-tátil, porém, mesmo com treinamento, podem ficar atrasados em comparação com os videntes, pois suas experiências táteis não compensam a visão na representação do corpo humano.

Tem sido demonstrado que adolescentes com visão residual apresentaram melhor conhecimento das partes do corpo do que aqueles com cegueira (PAPADOPOULOS; KOUSTRIAVA, 2011). Além disso, esses mesmos autores destacaram que adolescentes com deficiência visual enfrentaram dificuldades na lateralidade quando a referência era o outro e usaram estratégias alocêntricas para a representação do espaço próximo, obtendo desempenho equiparado ao de adolescentes vendados. Papadopoulos, Koustriava e Kartasidou (2012) relataram, entretanto, que a visão é fundamental para o desempenho nos processos de codificação e habilidades espaciais, consequentemente, a noção de corpo. Esses achados devem ser analisados com cautela, pois estudos apontam outros caminhos na formação do conceito corporal. Por exemplo, Witkin et al. (1968) demonstram que adolescentes com deficiência visual apresentaram bom desenvolvimento do conceito corporal a partir do teste de modelos de argila, considerando que há um esforço especial para a concepção de mundo, o que contribui para o desenvolvimento da diferenciação.

Nesse mesmo sentido, Duran e Tufenkjian (1969) demonstraram que adolescentes com deficiência visual utilizam o corpo como forma de medição. Em complemento, Jones, Taylor e Broadwell (2008) destacaram que a habilidade para medições que utilizavam o corpo pode ser mais acurada em adolescentes com deficiência da visão, pois demonstraramse mais precisos. Isso porque informações captadas pelo tato são importantes na construção 
de proporção, de lateralidade, do esquema e da imagem corporal nessa população. Koustriava e Papadopoulos (2012) demonstraram que há uma correlação positiva do conhecimento corporal e espacial com a direcionalidade e tomada de perspectiva nessa população, constatando que atrasos em habilidade espaciais podem estar associados ao desenvolvimento incompleto de outras habilidades espaciais, e não somente da deficiência visual. Em conjunto, esses achados demonstram que essa população pode desenvolver o conceito corporal adequadamente desde que receba estímulos apropriados. Com base nesses achados, a seguir apresenta-se a importância da atividade física na formação da imagem corporal.

\subsubsection{Atividade física/saúde}

Essa categoria abarcou estudos relacionados com a inatividade física como um fator que pode interferir negativamente no desenvolvimento da imagem corporal de adolescentes com deficiência visual (GREGUOL; GOBBI; CARRARO, 2014; YANG et al., 2016; HAEGELE; ZHU, 2018; CHEIKH; KASMI; MEHIDI, 2018), a relação entre a obesidade e adolescentes com deficiência visual (WRZESINSKA et al., 2016, 2017) e a prática de atividade física associada às autopercepções positivas (ANDERSON et al., 2019).

Em estudo que comparou adolescentes com deficiência visual no Brasil e na Itália, Greguol, Gobbi e Carraro (2014) demonstraram que, quanto maior a prática de atividade física, mais positiva a imagem corporal nessa população em ambos os países, entretanto a imagem corporal negativa pode aumentar o isolamento social e dificultar a participação em atividades físicas. Estratégias no sentido de aumentar a prática de atividade física dessa população são necessárias, no sentido de proporcionar um estilo de vida saudável (GREGUOL; GOBBI; CARRARO, 2014). Em consonância com esses achados, YANG et al., (2016) constataram que adolescentes com deficiência visual têm níveis mais baixos de atividade física em comparação com videntes e que o índice de massa corporal elevado está diretamente associado à prevalência da deficiência visual. Problemas oftalmológicos que surgem na infância, se tratados com intervenções precoces, podem aumentar a participação em aulas de Educação Física, favorecendo a imagem corporal positiva (YANG et al., 2016).

No mesmo sentido, o estudo de Cheikh, Kasmi e Mehidi (2018) também sinalizou que adolescentes com deficiência da visão têm um nível mais baixo de atividade física em comparação com videntes. Para os autores, uma possível explicação é que a deficiência pode 
QUINTANILHA, A.; GUTIERRES FILHO, P.; NEVES, C. M.; MEIRELES, J. FERREIRA, M. E. C.; SILVA, R. C. P. C. da; ALMEIDA, V. A. R. de; MORGADO, F. F. da R.

impactar o desempenho corporal, por isso esses autores reforçam a importância da atividade física para preservar a saúde desses adolescentes.

Outros achados demonstraram que adolescentes do sexo masculino com deficiência visual e alguma comorbidade estavam em maior risco de obesidade abdominal do que aqueles apenas com deficiência visual (WRZESINSKA et al., 2017). Constatou-se que a deficiência visual diminui a participação em atividades físicas, podendo ser um fator de risco para a obesidade, pois foram encontrados mais de $21 \%$ dos adolescentes com deficiência visual acima do peso e cerca de $15 \%$ eram obesos. Demonstrou-se, portanto, que a prevalência de excesso de peso e obesidade abdominal era mais comum em adolescentes com deficiência visual do que em seus pares sem essa deficiência (WRZESINSKA et al., 2016). Dessa forma, ações que promovam a participação desses alunos na prática de atividades físicas são necessárias.

Haegele e Zhu (2018), por exemplo, reportaram que o adolescente com deficiência visual pode desenvolver crenças de que a prática da Educação Física é desafiadora e incompatível com sua deficiência, o que influencia o desenvolvimento da imagem corporal negativa. Algo que chama a atenção nesse estudo é o fato de que professores de Educação Física excluíam os adolescentes com deficiência visual das aulas, reforçando o sentimento de limitação devido à deficiência. Esses dados demonstram que contextos de educação física escolar interferem no desenvolvimento de uma imagem corporal positiva ou negativa. Dessa forma, torna-se importante a implementação de aulas de Educação Física inclusivas para o desenvolvimento da imagem corporal positiva em adolescentes com deficiência da visão (WRZESINSKA et al., 2016; HAEGELE; ZHU, 2018).

Anderson et al. (2018) encontraram que a prática de atividade física entre adolescentes com deficiência visual promoveu saúde, condicionamento físico e vitalidade, gerando autopercepções positivas. Vale ressaltar que, nas aulas de Educação Física escolar, atividades pedagógicas propostas deveriam ser adaptadas com o intuito de incluir todos os alunos, oferecendo oportunidades para experimentar atividades variadas e acessíveis a diferentes condições individuais (MORGADO et al., 2017). Diante do exposto, pode-se compreender a importância da prática de atividade física, uma vez que pode intervir positivamente na constituição de uma imagem corporal integrada e positiva. Nesse mesmo caminho, buscou-se entender a deficiência visual relacionada com a insatisfação com o próprio corpo, o que será abordado a seguir. 


\subsection{Instrumentos de avaliação da imagem corporal e insatisfação corporal}

A presente categoria apresenta estudos que criaram instrumentos de avaliação da imagem corporal (MORGADO et al., 2013, 2014), bem como avaliaram a insatisfação corporal entre adolescentes com deficiência visual (ASHIKALI; DITTIMAR, 2010; PERES et al., 2015). Pesquisas que buscam desenvolver formas de avaliação da imagem corporal tiveram um importante crescimento nas últimas duas décadas, especialmente no Brasil (NEVES; MORGADO; TAVARES, 2015). Para adolescentes com deficiência visual, esse cenário não tem sido tão otimista. Somente dois estudos com criação de escalas na área foram encontrados. O primeiro foi o estudo de criação de uma escala tridimensional para avaliar a insatisfação corporal na população com cegueira congênita (Three-dimensional Body Rating Scale [3BRS]). Constatou-se que essa escala possui propriedades psicométricas adequadas em sua versão feminina, mas não na versão masculina (MORGADO et al., 2013).

Nesse mesmo caminho, outro estudo criou e validou a Escala de Autoaceitação para Pessoas com Cegueira Precoce (SAS-EB), incluindo adolescentes com cegueira precoce entre 18 e 19 anos. A SAS-EB mostrou-se um instrumento confiável e válido para medir a autoaceitação nessa parcela da população (MORGADO et al., 2014). Diante da escassez de escalas e da demanda por avaliar a imagem corporal de pessoas com deficiência visual, a criação e validação de instrumentos para essa população são fundamentais. Vale ressaltar que pesquisas que visem a desenvolver instrumentos de avaliação exigem conhecimento teórico consistente, planejamento cuidadoso, rigor na coleta de dados, interpretação de dados que não considere apenas provas matemáticas (no caso de pesquisas quantitativas), compreensão do constructo a partir das evidências geradas pelos dados, bem como a capacidade de articular o que é relatado pelo sujeito com as evidências já estabelecidas nesse campo de pesquisa (THOMPSON, 2004; NEVES; MORGADO; TAVARES, 2015). No âmbito da insatisfação corporal, Ashikali e Dittimar (2010) relataram que mulheres com cegueira congênita apresentam menores níveis de insatisfação corporal em comparação com mulheres com cegueira adquirida e videntes. Possivelmente, isso se dá pelo fato de não verem padrões de beleza impostos pela mídia visual. Esses achados se coadunam com uma pesquisa que verificou que, quanto menor o comprometimento visual, maior a insatisfação corporal (PERES et al., 2015). Dessa forma, parece que pessoas com deficiência visual congênita apresentam menores níveis de insatisfação corporal. 
QUINTANILHA, A.; GUTIERRES FILHO, P.; NEVES, C. M.; MEIRELES, J. FERREIRA, M. E. C.; SILVA, R. C. P. C. da; ALMEIDA, V. A. R. de; MORGADO, F. F. da R.

Em síntese, a presente categoria demonstrou que há acentuada escassez de instrumentos válidos e confiáveis para avaliar a imagem corporal de adolescentes com deficiência visual. Futuras pesquisas na área poderiam realizar a adaptação transcultural e a validação psicométrica de instrumentos de medidas especificamente para essa população ou, ainda, criar instrumentos específicos para essa finalidade. Assim, seria possível conhecer de modo sistemático o desenvolvimento da imagem corporal nessa população, bem como acompanhar possíveis programas de intervenção.

Algumas limitações podem ser ressaltadas. Os estudos incluídos possuem informações variadas em relação a idade dos participantes, objetivos de pesquisa, grau de deficiência visual, contextos culturais, método e medidas de pesquisa, o que dificultou a comparação dos estudos e resultados entre si. Outra questão é que alguns estudos incluídos nessa revisão abrangiam uma parte da faixa etária considerada elegível. Nesse caso, os resultados refletiram, em sua grande parte, escores para outra idade, o que é entendido como limitação. Outro ponto é que, durante a busca dos artigos nas bases de dados, utilizaram-se all fields, acarretando que, em alguns artigos, essa expressão fosse encontrada nas referências. Alguns estudos, portanto, não relacionam imagem corporal diretamente com adolescentes com deficiência visual, porém foram incluídos, por terem relação indireta com a imagem corporal e também devido à escassez de estudos.

\section{Conclusão}

A presente revisão sistemática buscou demonstrar evidências científicas da imagem corporal de adolescentes com deficiência visual. Na maioria dos estudos, foram encontradas implicações negativas da deficiência visual na formação de uma imagem corporal positiva nessa população, contudo o prognóstico desfavorável para adolescentes com deficiência visual muitas vezes se baseia nas limitações da deficiência, valorizando o déficit orgânico. A temática abordada neste estudo não tem por objetivo negar a deficiência visual enquanto uma limitação que exige adequações, no entanto podem-se questionar resultados que se pautam apenas nos atrasos físicos, cognitivos e psicossociais do adolescentes com deficiência da visão. Futuras pesquisas podem se debruçar em entender aspectos específicos da deficiência visual em adolescentes, como, por exemplo, formação da imagem corporal no contexto de inclusão escolar e mudanças corporais da puberdade nessa população. Dessa forma, seria 
possível ampliar a compreensão das necessidades desses jovens, provendo conhecimento aplicado aos processos socioemocionais que desencadeiam a imagem corporal positiva.

Este projeto foi financiado pela Fundação de Amparo à Pesquisa do Estado do Rio de Janeiro (FAPERJ).

\section{Referências}

ABBOTT, B. D.; BARBER, B. L. Embodied image: Gender differences in functional and aesthetic body image among Australian adolescents. Body Image v. 7, p. 22-31, 2010.

ALEXIAS, G.; TOGAS, C.; MELLON, R. Psychometric properties of the Greek version of the body appreciation scale. Hellenic Journal of Psychology, v. 13, 2016.

ANDERSON, R.; WARREN, N.; MISAJON, R.; LEE DPSYCH, S. Exploring Wellbeing in Youth with Vision Impairment: Insights for Vision Rehabilitation. Applied Research in Quality of Life, 2019.

ANDERSON, R.; WARREN, N.; MISAJON, R.; LEE DPSYCH, S. You need the more relaxed side, but you also need the adrenaline: promoting physical health as perceived by youth with vision impairment. Disability and Rehabilitation, 2018.

ARGYRIDES, M.; KKELI, N. Validation of the factor structure of the Greek adaptation of the Situational Inventory of Body-Image Dysphoria. Eating and Weight Disorders, Dec. v. 20, n. 4, p. 491-6, 2015.

ASHIKALI, E. M.; DITTIMAR, H. Body image and restrained eating in blind and sighted Women: A preliminary study. Body Image, 2010.

BARDIN, L. Análise de conteúdo. Tradução de Luís Antero Retos, Augusto Pinheiro. São Paulo: Edições 70, 2016.

BISOL, C. A.; PEGORINI, N. N.; VALENTINI, C. B. Pensar a deficiência a partir dos modelos médico, social e pós-social. Cad. Pes., São Luís, v. 24, n. 1, jan./abr., 2017.

BLAKEMORE, S. J.; MILLS, K. Is Adolescence a Sensitive Period for Sociocultural Processing? Rev. Psychol, v. 65, p. 187-207, 2014.

BONOTIS, K.; PANTELIS, K.; KARAOULANIS, S.; ZAFIRIOU, E.; TSOGAS, P. Investigation of factors associated with health-related quality of life and psychological distress in vitiligo. Journal of the German Society of Dermatology, v. 14, n. 1, p. 45-9, 2016.

BOURNE. R. R., FLAXMAN, S. R., BRAITHWAITE, T., CICINELLI, M. V., DAS, A., JONAS, J. B.; ZHENG, Y. Magnitude, temporal trends, and projections of the global prevalence of blindness and distance and near vision impairment: a systematic review and meta-analysis, Vision \& Eye Research Unit, 2017. 
QUINTANILHA, A.; GUTIERRES FILHO, P.; NEVES, C. M.; MEIRELES, J. FERREIRA, M. E. C.; SILVA, R. C. P. C. da; ALMEIDA, V. A. R. de; MORGADO, F. F. da R.

CASH, T. et al. Measuring "negative body image": Validation of the Body Image

Disturbance Questionnaire in a nonclinical population. Body Image, v. 1, n. 4, p. 363-372, 2004.

CHEIKH, Y.; KASMI, B.; MEHIDI, M. The level of health-related fitness of male blind versus sighted pupils. Gazzetta Medica Italiana, 2018.

DAVISON, T. E.; MCCABE, M. P. Adolescent Body Image and Psychosocial Functioning. The Journal of Social Psychology, v. 146, n. 1, p. 15-30, 2006.

DURAN, P.; TUFENKJIAN, S. Tactile-kinesthetic methods for measuring length used by congenitally blind children. Perceptual and Motor Skills, 1969.

FARHAT, T.; IANNOTTI, R. J.; CACCAVALE, L. J. Adolescent Overweight, Obesity and Chronic Disease-Related Health Practices: Mediation by Body Image. Obesity Facts, v. 7 , p.1-14, 2014.

FERNANDEZ, A. B.; PANZA, G. A.; CRAMER, B. D.; CHATTERJEE, S.E;

JAYARAMAN, R.F. Age-related macular degeneration and incident stroke: A systematic review and meta-analysis. PLoS ONE, v. 10, n. 11, e0142968, 2015.

FRANÇA, T. H. Lutas Sociais, Modelo Social da Deficiência: uma ferramenta sociológica para a emancipação social. São Paulo, v.17, n. 31, p. 59-73, jul./dez. 2013.

GAUDENSI, P.; ORTEGA, F. Problematizando o conceito de deficiência a partir das noções de autonomia e normalidade. Ciência \& Saúde Coletiva, v. 21, n. 10, p. 3061-3070, 2016.

GREGUOL, M.; GOBBI, E.; CARRARO, A. Physical activity practice, body image and visual impairment: A comparison between Brazilian and Italian children and Adolescents.

Research in Developmental Disabilities, v. 35, p. 21-26, 2014.

GÜLER, A. S.; ARMAN, A. R.; GENCER, A. G. Distorted body image in the presence of visual loss in a 15 year-old adolescents. Neurlogypsychiatry and Brain Research, 2012.

GZIL, F.; LEFEVE, C.; CAMMELLI, M.; PACHOUD, B.; RAVAUD, J. F.; LEPLEGE, A. Why is rehabilitation not yet fully person-centred and should it be more person-centred?

Disability and Rehabilitation, v. 29, n. 20-21, p. 1616-1624, 2007.

HALDER, S.; DATTA, P. An exploration into self-concept: A comparative analysis between the adolescents who are sighted and blind in India. The British Journal of Visual

Impairment, 2011.

HAEGELE, J.; ZHU, X. Body image and physical education: Reflections of individuals with visual impairments. European Physical Education Review, p. 1-15, 2018.

JALALI, M. S.; RAMANDAD, H.; BULLOCK, S. L.; LEE-KWAN, S. H.; GITTELSOHN, J.; AMMERMAN, A. Dynamics of intervention adoption, implementation, and maintenance inside organizations: The case of an obesity prevention initiative. Social Science $\boldsymbol{\&}$

Medicine, v. 224, p. 67-76, 2019. 
JENKS, E. B. Explaining disability: parents' stories of raising children with visual impairments in a sighted world. Journal of Contemporary Ethnography, v. 34, p. 143-169, 2005.

JONES, D. C. Body Image Among Adolescent Girls and Boys: A Longitudinal Study. Developmental Psychology, v. 40, n. 5, p. 823-835, 2004.

JONES, M. G.; TAYLOR, A. R.; BROADWELL, B. Concepts of Scale Held by Students With Visual Impairment. Journal of Research in Science Teaching, v. 46, n. 5, p. 506-519, 2008 .

JUDITH, W.; CAROL, G. Handbook of girls' and women's psychological health: Gender and well-being across the lifespan. Psychological Medicine, v. 36, n. 7, p. 1043-1044, 2006.

KAPLAN-MYRTH, N. Alice Without A Looking Glass: Blind People And Body Image. Anthropology \& Medicine, v. 7, n. 3, 2000.

KINSBOURNE, M.; LEMPERT, H. Human figure representation by blind children, Journal of general psychology, 1980.

KOCOURKOVA, J.; SOLTYSOVA, M.; MARKETA, M.; HRDLICKA, M. Anorexia nervosa in a blind girl. Neuroendocrinol Letters, 2011.

KOUSTRIAVA, E.; PAPADOPOULOS, K. Are there relationships among different spatial skills of individuals with blindness? Research in Developmental Disabilities, v. 33, p. 2164-2176, 2012.

LAUS, M. F.; KAKESHITA, I. S.; BRAGA-COSTA, T. M.; FERREIRA, M. E. C.; FORTES, L. S.; ALMEIDA, S. S. Body image in Brazil: Recent advances in the state of knowledge and methodological issues. Revista de Saúde Pública, v. 48, n 2, p. 331-346, 2014.

MÄKINEN, M.; MARTTUNEN, M.; KOMULAINEN, E.; TEREVNIKOV, V.;

VIERTOMIES, L. R. P.; AALBERG, V.; LINDBERG, N. Development of self-image and its components during a one-year follow-up in non-referred adolescents with excess and normal weight child and adolescent. Psychiatry and Mental Health, 2015.

MOHER, D.; LIBERATI, A.; TETZLAFF, J.; ALTMAN, D. The PRISMA Group, Preferred reporting items for systematic reviews and meta-analyses: The PRISMA Statement. PLoS Med, v. 6, 2008.

MORGADO, F. F. R.; CAMPANA, A. N. N. B.; FERREIRA, M. E. C.; RIGBY, A. S. Initial evidence of the reliability and validity Of a three-dimensional body rating scale for the congenitally blind. Perceptual \& Motor Skills, v. 116, n. 1, p. 91-105, 2013.

MORGADO, F. F. R.; CAMPANA, A. N. N. B.; TAVARES, M. C. G. C. F. Development and Validation of the Self-Acceptance Scale for Persons with Early Blindness: The SAS-EB. PLoS ONE, v. 9, n. 9, e106848, 2014.

MORGADO, F. R. R.; CASTRO, M., R.; FERREIRA, M. E. C.; OLIVEIRA, A. J.;

PEREIRA, J. G.; SANTOS, J. H. Representações Sociais sobre a Deficiência: Perspectivas de 
QUINTANILHA, A.; GUTIERRES FILHO, P.; NEVES, C. M.; MEIRELES, J. FERREIRA, M. E. C.; SILVA, R. C. P. C. da; ALMEIDA, V. A. R. de; MORGADO, F. F. da R.

Alunos de Educação Física Escolar. Rev. Bras. Ed. Esp., Marília, v. 23, n. 2, p. 245-260, abr./jun., 2017.

MORGADO, F. F. R.; NEVES, A. N.; FORTES, L. S.; FERNANDES, M. C. G. C. Implicações da Cegueira Congênita na Imagem Corporal: Uma Revisão Integrativa.

Psicologia: Teoria e Pesquisa, v. 35, e35415, 2019.

NEVES, A. N.; MORGADO, F. F. R.; TAVARES, M. C. G. C. F. Avaliação da imagem corporal: notas essenciais para uma boa prática de pesquisa. Psicologia: Teoria e Pesquisa, v. 31, n. 3, p. 375-380, jul./set., 2015.

OtTAiano, J. A. A., ÁVIlA, M. P., UMBElinO, C. C., TALEB, A. C. As condições de saúde ocular no Brasil. Conselho Brasileiro de Oftalmologia, 2019.

PAGE, A.; PAPPS, F. A. Eating Attitudes of Women Living With a Vision Impairment. Psychology of Women Quarterly, v. 42, n. 4, p. 477-488, 2018.

PAPADOPOULOS, K.; KOUSTRIAVA, E. The impact of vision in spatial coding. Research in Developmental Disabilities, v. 32, p. 2084-2091, 2011.

PAPADOPOULOS, K.; KOUSTRIAVA, E.; KARTASIDOU, L. Spatial coding of individuals with visual impairments. The Journal of Special Education, 2012.

PAPADOPOULOS, K.; KOUSTRIAVA, E.; KARTASIDOU, L. The Impact of Residual Vision in Spatial Skills of Individuals With Visual Impairments. The Journal of Special Education, v. 45, n. 2, p. 118-127, 2011.

PERES, R. J.; ESPIRITO SANTO, G.; ESPIRITO, F. R.; FERREIRA, N. T.; ASSIS, M. R. Insatisfação com a imagem corporal entre pessoas com deficiência visual, Rev Bras Ciênc Esporte, 2015.

PIERCE, J. W.; WARDLE, J. Body Size, Parental Appraisal, and Self-Esteem in Blind Children. J. Child Psychol. Psychial, 1996.

PINQUART, M.; PFEIFFER, J. P. Body image in adolescents with and without visual impairment. The British Journal of Visual Impairment, 2012.

PINQUART, M.; PFEIFFER, J. P. Does visual impairment lead to lower or higher levels of success in solving developmental tasks? J. Dev. Phys Disabil., 2013a.

PINQUART, M.; PFEIFFER, J. P. Perceived social support in adolescents with and without visual impairment. Research in Developmental Disabilities, $2013 \mathrm{~b}$.

RIBEIRO, L. O. M., A inclusão do aluno com deficiência visual em contexto escolar: afeto e práticas pedagógicas. Revista Educação, Artes e Inclusão, 2017.

SCHILDER, P. A imagem do corpo. 2. ed. São Paulo: Martins Fontes, 1994.

SCHIPPER, T.; LIEBERMAN, L. J.; MOODY, B. "Kids like me, we go lightly on the head": Experiences of children with a visual impairment on the physical self-concept. British Journal of Visual Impairment, v. 35, n. 1, p. 55-68, 2017. 
SIRRIYEH, R.; LAWTON, R.; GARDNER, P.; ARMITAGE, G. Reviewing studies with diverse designs: The development and evaluation of a new tool. Journal of Evaluation in Clinical Practice, v. 18, p. 746-752, 2012.

SOULLIARD, J. S.; WAL, J. S. V. Validation of the Body Appreciation Scale-2 and relationships to eating behaviors and health among sexual minorities. Body Image, v. 31, p. 120-130, 2019.

STAMER, W. D.; SCHUMAN, J. S. Introduction to Gedenkschrift for David L. Epstein. Experimental Eye Research, v. 158, 2017.

THOMPSON, J. K.; VAN DEN BERG, P.; ROEHRIG, M.; GUARDA, A. S.; HEINBERG, L. J. The Sociocultural Attitudes towards Appearance Scale (SATAQ-3): Development and validation. International Journal of Eating Disorders, v. 35, p. 293-304, 2004.

THOMPSON, J. K.; BURKE, N. L.; KRAWCZYK, R. Mesurement of body image in adolescence and adulthood. In: CASH, T. F. (ed.). Encyclopedia of body image andhuman appearance, v. 2, p. 512-520. San Diego, CA: AcademicPress/Elsevier, 2012.

TREMBLAY, L. C.; ROY-VAILLANCOURT, M.; CHEBBI, B.; BOUCHARD, S.; DAOUST, M.; DENOMMEE, J.; THORPE, M. Body Image and Anti-Fat Attitudes: An Experimental Study Using a Haptic Virtual Reality Environment to Replicate Human Touch. Cyberpsychology, behavior, and social networking, v. 19, n. 2, p. 100-6, 2016.

TYLKA, T. L.; WOOD-BARCALOW. N. L. The Body Appreciation Scale-2: Item refinement and psychometric evaluation. Body Image, v. 12, 2015.

VANDEREYCKEN, W. Anorexia nervosa and visual impairment. Comprehensive Psychiatry, 1986.

WANG, S. B.; HAYNOS, A. F.; WALL, M. M.; CHEN, C.; EISENBERG, M. E.; UMARKSZTAINER, D. Fifteen-Year Prevalence, Trajectories, and Predictors of Body Dissatisfaction From Adolescence to Middle Adulthood. Clinical Psychological Science, v. 7, n. 6, p. 1403 1415, 2019.

WATEMBERG, J.; SERMARK, S.A.; HENDERSON, A. Right-Left Discrimination in Blind and Sighted Children. Physical \& Occupational Therapy in Pediatrics, 1986.

WITKIN, H. A.; BIRNBAUM, J.; LOMONACO, S., LEHR, S.; HERMAN, J. L. Cognitive Patterning in Congenitally Totally Blind Children. Child Development, 1968.

WHO. Women and health: today's evidence tomorrow's agenda, 2009.

WRZESINSKA, M.; URZEDOWICZ, B.; MOTYLEWSK, S.; ZEMAN, K.; PAWLICKIL, L. Body mass index and waist-to-height ratio among schoolchildren with visual impairment. Medicine, v. 95, n. 32, 2016.

WRZESINSKA, M.; URZEDOWICZ, B.; NAWARYCZ, T., MOTYLEWSK, S.; PAWLICKIL, L. The prevalence of abdominal obesity among pupils with visual impairment in Poland. Disability and Health Journal, v. 10 p. 559-564, 2017. 
QUINTANILHA, A.; GUTIERRES FILHO, P.; NEVES, C. M.; MEIRELES, J. FERREIRA, M. E. C.; SILVA, R. C. P. C. da; ALMEIDA, V. A. R. de; MORGADO, F. F. da R.

YANG, F.; YANG, C.; LIU, Y.; PENG, S.; LIU, B.; GAO, X.; TAN, X. Associations between Body Mass Index and Visual Impairment of Schools students in Central China. Int. J. Environ. Res. Public Health, 2016.

Enviado em: 02/07/2020

Revisado em: 18/01/2021

Aprovado em: 04/02/2021

Publicado em: 15/06/2021 